\title{
Letter
}

\section{Hydrodynamic fluctuations from a weakly coupled scalar field}

\author{
G. Jackson ${ }^{\mathrm{a}}$, M. Laine ${ }^{\mathrm{b}}$ \\ AEC, Institute for Theoretical Physics, University of Bern, Sidlerstrasse 5, 3012 Bern, Switzerland
}

Received: 8 March 2018 / Accepted: 8 April 2018 / Published online: 18 April 2018

(C) The Author(s) 2018

\begin{abstract}
Studies of non-equilibrium dynamics of firstorder cosmological phase transitions may involve a scalar field interacting weakly with the energy-momentum tensor of a thermal plasma. At late times, when the scalar field is approaching equilibrium, it experiences both damping and thermal fluctuations. We show that thermal fluctuations induce a shear viscosity and a gravitational wave production rate, and propose that including this tunable contribution may help in calibrating the measurement of the gravitational wave production rate in hydrodynamic simulations. Furthermore it may enrich their physical scope, permitting in particular for a study of the instability of growing bubbles.
\end{abstract}

\section{Introduction}

With the planning of the LISA interferometer under way, it has become timely to consider gravitational wave production from cosmological phase transitions [1]. This process is dominated by non-equilibrium sources, with a considerable contribution originating from a late stage with sound waves and/or turbulence (cf. Ref. [2] for an overview of recent work). Eventually this motion terminates and the system reaches thermal equilibrium. In the equilibrium state the production of gravitational waves continues through thermal fluctuations [3] but the magnitude of this component is in general much below the observable level.

A phase transition proceeds through the nucleation and subsequent growth, collisions, and coalescence of bubbles of the low-temperature phase. As they are growing, the bubble walls reach a steady velocity, because of friction (cf. Ref. [4] and references therein). Friction can be represented by a dissipative coefficient in the equation of motion for the order parameter of the transition ("scalar field"). The fluctuationdissipation theorem asserts that dissipation implies the pres-

\footnotetext{
a e-mail: jackson@itp.unibe.ch

b e-mail: laine@itp.unibe.ch
}

ence of fluctuations. The purpose of this study is to show how thermal fluctuations of the scalar field can be included in a framework frequently used for numerical simulations [2], and which physical influence they may be expected to have there.

Before proceeding to the scalar field case, let us note that the case of "normal" hydrodynamic fluctuations is for completeness briefly reviewed in Appendix A.

\section{Hydrodynamics coupled to a scalar field}

\subsection{Original setup}

We start by recapitulating the basic equations without fluctuations. For generality the system is put in a curved background with a metric $g^{\mu \nu}$, even if for some considerations it is sufficient to subsequently restrict to the Minkowski metric or to linear perturbations around it.

As the basic variables, we adopt a real scalar field $\phi$ and a plasma parametrized by a local temperature $T$ and a flow velocity $u^{\mu}$. The energy-momentum tensor is postulated to have the form

$$
\begin{aligned}
T^{\mu \nu} & \equiv \phi^{, \mu} \phi^{, v}-\frac{g^{\mu v} \phi_{, \alpha} \phi^{, \alpha}}{2}+w u^{\mu} u^{v}+p g^{\mu v} \\
p & \equiv p_{0}(T)-V(\phi, T), \quad w \equiv T \partial_{T} p
\end{aligned}
$$

where $p$ is the pressure, $w$ is the enthalpy density, and $u^{\mu}$ is the flow velocity. By ()$_{, \mu}$ we denote a partial derivative in the $x^{\mu}$-direction, whereas ()$_{; \mu}$ is a covariant derivative. For $g^{\mu \nu}$ we employ the "mostly plus" metric convention, so that $u_{\mu} u^{\mu}=-1$. Within a derivative expansion (slow variations) the basic equations are [5]

$$
\begin{aligned}
T_{; \mu}^{\mu \nu} & =0, \\
\phi_{; \mu}^{; \mu}-\gamma u^{\mu} \phi_{, \mu}-\partial_{\phi} V & =0 .
\end{aligned}
$$


The coefficient $\gamma$ parametrizes entropy production in regions where the scalar field varies (i.e. particularly around bubble walls): $T\left(s u^{\mu}\right)_{; \mu}=\gamma\left(u^{\mu} \phi_{, \mu}\right)^{2}$, where $s \equiv \partial_{T} p$.

Without the scalar field contribution, the energymomentum tensor would be that of an ideal fluid. In that system phase transition fronts and shocks appear as discontinuities [6]. Originally, the introduction of $\phi$ was motivated by having a microscopically adjustable parametrization of the entropy production that takes place at these discontinuities [5]. However, lately the same model is also used for studying the subsequent stages with more complicated dynamics [2]. With many overlapping sound waves, the system starts to resemble a thermal plasma with various random motions taking place simultaneously.

We note in passing that we do not consider here the microscopic origin of the coefficient $\gamma$. In general it is a function of $\phi$, though it is expected to have a non-zero value even as $\phi \rightarrow 0$ [7]. In the following we are concerned with the "final state" of the system, which in the context of the electroweak phase transition means the low-temperature Higgs phase, $\phi \approx \phi_{0}(T)$. We shift $\phi$ by $\phi_{0}(T)$ so that after the shift $V(\phi, T) \equiv \frac{1}{2} m^{2}(T) \phi^{2}$, and ignore scalar field selfinteractions. Terms originating from the shift by $\phi_{0}(T)$ have been included in $p_{0}(T)$.

The equations presented above should apply in the socalled hydrodynamic regime [8], i.e. at time and length scales $\gtrsim 1 /\left(\alpha^{2} T\right)$, where $\alpha$ is a coupling characterizing the interactions within the plasma. At the electroweak epoch $T \sim 100 \mathrm{GeV}$, and the Hubble radius is $H^{-1} \sim 10^{15} T^{-1}$. The bubble distance scale is a macroscopic fraction of the latter, say $10^{-2} H^{-1}$ [2], and thus indeed huge compared with $1 /\left(\alpha^{2} T\right)$, even if $\alpha \sim 10^{-2}$.

\subsection{Thermal fluctuations}

Whenever dissipation is present, the fluctuation-dissipation theorem needs to be respected [8]. This implies that the scalar field equation in Eq. (2.4) should actually be corrected into

$\phi_{; \mu}^{; \mu}-\gamma u^{\mu} \phi_{, \mu}-m^{2} \phi+\xi=0$,

where $\xi$ is a stochastic noise term. The autocorrelator of the noise is assumed to take the form

$\langle\xi(\mathcal{X}) \xi(\mathcal{Y})\rangle=\frac{\Omega \delta(\mathcal{X}-\mathcal{Y})}{\sqrt{-\operatorname{det} g_{\mu \nu}}}, \quad \mathcal{X}=(t, \mathbf{x})$,

where $\Omega$ is a coefficient whose value is determined presently (cf. Eq. (2.12))

Let us solve Eq. (2.5) in local Minkowskian coordinates $\left(g_{\mu \nu} \rightarrow \eta_{\mu \nu}=(-+++)\right)$ in a domain of a constant 4-velocity $u^{\mu}$. Considering times $\gg \gamma^{-1}$ so that initial transients have died out, the solution can be written as

$$
\begin{aligned}
\phi(\mathcal{X}) & =\int_{\mathcal{Y}} G_{\mathrm{R}}(\mathcal{X}-\mathcal{Y}) \xi(\mathcal{Y}) \\
G_{\mathrm{R}}(\mathcal{X}) & =\int_{\mathcal{P}} \frac{e^{i \mathcal{P} \cdot \mathcal{X}}}{\mathcal{P}^{2}+i \gamma \mathcal{P} \cdot u+m^{2}}
\end{aligned}
$$

where $\mathcal{P}=(\omega, \mathbf{p})$ and $\int_{\mathcal{P}} \equiv \int_{-\infty}^{\infty} \frac{\mathrm{d} \omega}{2 \pi} \int \frac{\mathrm{d}^{d} \mathbf{p}}{(2 \pi)^{d}}$. For $\gamma>0$ the poles in Eq. (2.8) are in the lower half-plane, and therefore $G_{\mathrm{R}}(\mathcal{X})$ is a retarded Green's function. Making use of the autocorrelator in Eq. (2.6) the 2-point function becomes

$$
\begin{aligned}
\langle\phi(\mathcal{X}) \phi(\mathcal{Y})\rangle & =\Omega \int_{\mathcal{Z}} G_{\mathrm{R}}(\mathcal{X}-\mathcal{Z}) G_{\mathrm{R}}(\mathcal{Y}-\mathcal{Z}) \\
& =\int_{\mathcal{P}} \frac{\Omega e^{i \mathcal{P} \cdot(\mathcal{X}-\mathcal{Y})}}{\left(\mathcal{P}^{2}+m^{2}\right)^{2}+\gamma^{2}(\mathcal{P} \cdot u)^{2}} .
\end{aligned}
$$

We thus see that, in analogy with the real-time formalism of thermal field theory [9], Feynman rules for this system contain two types of propagators, the retarded propagator $G_{\mathrm{R}}$ and a statistical propagator $\langle\phi \phi\rangle$ originating from the average $G_{\mathrm{R}}\langle\xi \xi\rangle G_{\mathrm{R}}$.

The integral over $\omega$ can be carried out in Eq. (2.9). In particular, setting the time arguments equal and denoting $\epsilon_{p}^{2} \equiv p^{2}+m^{2}$ with $p \equiv|\mathbf{p}|$, we get

$$
\begin{aligned}
\langle\phi(t, \mathbf{x}) \phi(t, \mathbf{y})\rangle= & \frac{\Omega}{2 \gamma} \int_{\mathbf{p}} \frac{e^{i \mathbf{p} \cdot(\mathbf{x}-\mathbf{y})}}{2 \epsilon_{p}} \\
& \times\left\{\frac{1}{\epsilon_{p} u^{0}-\mathbf{p} \cdot \mathbf{u}}+\frac{1}{\epsilon_{p} u^{0}+\mathbf{p} \cdot \mathbf{u}}\right\} .
\end{aligned}
$$

In order to fix the value of $\Omega$, let us compare Eq. (2.10) with the direct computation of the 2-point correlator in an ensemble defined by the density matrix $\hat{\rho} \equiv Z^{-1} e^{-\left(\hat{H} u^{0}-\hat{K}^{i} u^{i}\right) / T}$, where $Z$ is the partition function, $\hat{H}$ is the Hamiltonian, and $\hat{K}^{i}$ is the momentum operator. We obtain

$$
\begin{aligned}
& \operatorname{Tr}\{\hat{\rho} \hat{\phi}(t, \mathbf{x}) \hat{\phi}(t, \mathbf{y})\}=\int_{\mathbf{p}} \frac{e^{i \mathbf{p} \cdot(\mathbf{x}-\mathbf{y})}}{2 \epsilon_{p}} \\
& \quad \times\left\{1+n_{\mathrm{B}}\left(\epsilon_{p} u^{0}-\mathbf{p} \cdot \mathbf{u}\right)+n_{\mathrm{B}}\left(\epsilon_{p} u^{0}+\mathbf{p} \cdot \mathbf{u}\right)\right\},
\end{aligned}
$$

where $n_{\mathrm{B}}(x) \equiv 1 /[\exp (x / T)-1]$ is the Bose distribution. The hydrodynamic description of Eq. (2.10) is supposed to apply for $\epsilon_{p}, p \ll T$. Expanding $n_{\mathrm{B}}(x) \approx T / x$ and comparing Eqs. (2.10) and (2.11), we uniquely identify the noise autocorrelator $\Omega$ as

$\Omega=2 \gamma T$.

More generally, if $\phi$ is not in equilibrium with the medium, the $T$ in Eq. (2.12) could differ from that in Eq. (2.2). 


\subsection{Energy-momentum correlator}

Let us now define a "transverse-traceless" (TT) correlator of the energy-momentum tensor $T^{\mu \nu}$, after choosing the spatial momentum to point in the $z$-direction:

$$
C_{\Delta}^{\mathrm{TT}}\left(k^{0}, k\right) \equiv \int_{\mathcal{X}} e^{i k^{0} t-i k z}\left\langle\frac{1}{2}\left\{T^{x y}(t, \mathbf{x}), T^{x y}(0)\right\}\right\rangle .
$$

With the classical fields that appear in hydrodynamics, operator ordering plays actually no role. For convenience we denote the infrared limit of this correlator by

$$
\lim _{k^{0}, k \rightarrow 0} C_{\Delta}^{\mathrm{TT}}\left(k^{0}, k\right) \equiv 2 \eta T
$$

Through a standard Kubo relation, $\eta$ can be interpreted as an effective overall "shear viscosity" of the coupled system (fluid $+\phi$ ), but for the purposes of the present paper Eq. (2.14) can equally well be taken as a definition of $\eta$. In any case, the infrared contribution to the differential production rate of the energy density carried by gravitational waves reads [3]

$\lim _{k \rightarrow 0} \frac{\mathrm{d} e_{\mathrm{GW}}}{\mathrm{d} t \mathrm{~d}^{3} \mathbf{k}}=\frac{4 \eta T}{\pi^{2} m_{\mathrm{Pl}}^{2}}$,

where $m_{\mathrm{Pl}}=1.22 \times 10^{19} \mathrm{GeV}$ is the Planck mass.

Rather than directly computing the correlator in Eq. (2.13), which could be achieved through the use of Eq. (2.9), it is illuminating to couple the system to a metric perturbation $h_{x y}(t, z) \equiv h_{x y} e^{-i k^{0} t+i k z}$. The response of the expectation value of $T^{x y}$ to this background yields the retarded correlator [10], from which the time ordering in Eq. (2.13) can be readily extracted (assuming that the scalar field is in thermal equilibrium à la Eq. (2.12)):

$C_{\Delta}^{\mathrm{TT}}\left(k^{0}, k\right)=\left[1+2 n_{\mathrm{B}}\left(k^{0}\right)\right] \lim _{h_{x y} \rightarrow 0} \operatorname{Im}\left[\delta\left\langle T^{x y}\right\rangle / \delta h_{x y}\right]$.

Working to leading order in small perturbations $\left(\phi, u^{i}\right)$ and to linear order in $h_{x y}$, Eq. (2.5) takes the form

$$
\left(\partial_{t}^{2}+\gamma \partial_{t}-\nabla^{2}+m^{2}\right) \phi=\xi-2 h_{x y} \phi_{, x, y}+\mathcal{O}\left(h_{x y}^{2}, \phi u^{i}\right) .
$$

This can be solved as

$$
\begin{aligned}
\phi(\mathcal{X}) \approx & \int_{\mathcal{Y}} G_{\mathrm{R}}(\mathcal{X}-\mathcal{Y}) \xi(\mathcal{Y}) \\
& -2 \int_{\mathcal{Y}, \mathcal{Z}} G_{\mathrm{R}}(\mathcal{X}-\mathcal{Y}) h_{x y}(\mathcal{Y}) G_{\mathrm{R}, x, y}(\mathcal{Y}-\mathcal{Z}) \xi(\mathcal{Z})
\end{aligned}
$$

Inserting into $\left\langle T_{\phi}^{x y}\right\rangle \equiv\left\langle\phi_{, x} \phi_{, y}\right\rangle$, averaging over fluctuations, integrating over energy, and omitting terms suppressed by $k^{2} / \gamma^{2}$, we obtain

$$
\left\langle T_{\phi}^{x y}\right\rangle \approx T h_{x y}(t, z) \int_{\mathbf{p}} \frac{p_{x}^{2} p_{y}^{2}}{\epsilon_{p}^{4}} \frac{1+\frac{i \gamma}{k^{0}+i \gamma}}{1-\frac{k^{0}\left(k^{0}+2 i \gamma\right)}{4 \epsilon_{p}^{2}}} .
$$

For fixed $k^{0} / \gamma$ and to leading order in $\gamma^{2} / \epsilon_{p}^{2}$, we thus find a Lorentzian shape $1+\frac{i \gamma}{k^{0}+i \gamma}$ for the retarded correlator.

\subsection{Ultraviolet problem and finite part}

Inserting Eq. (2.19) into Eqs. (2.14) and (2.16) yields a scalar contribution to the effective shear viscosity,

$\delta \eta=\frac{T}{\gamma} \int_{\mathbf{p}} \frac{p_{x}^{2} p_{y}^{2}}{\left(p^{2}+m^{2}\right)^{2}}\left(1+\frac{\gamma^{2}}{p^{2}+m^{2}}\right)$.

The same result can be obtained from a direct computation of the correlator in Eq. (2.13), along lines illustrated for normal hydrodynamic fluctuations in Appendix A.

As is familiar from classical field theory [11,12], the result is power-divergent at large momenta. Cutting off large momenta so that $p \leq \Lambda$, the divergent part reads

$\left.\delta \eta\right|_{\mathrm{div}}=\frac{T}{90 \pi^{2} \gamma}\left[\Lambda^{3}+3\left(\gamma^{2}-2 m^{2}\right) \Lambda\right]$.

If the theory is rather regularized on a (comoving) lattice, so that the autocorrelator in Eq. (2.6) becomes $\Omega \delta(\mathcal{X}-\mathcal{Y}) /$ $\sqrt{-\operatorname{det} g_{\mu \nu}} \rightarrow \Omega \delta_{x^{0}, y^{0}} \delta_{\mathbf{x}, \mathbf{y}} /\left(a_{t} a_{s}^{3}\right)$, where $a_{t}$ and $a_{s}$ are the temporal and spatial lattice spacings, respectively, partial integration and rotational invariance permit to reduce the cubic divergence to a known tadpole $[13,14]$,

$$
\begin{aligned}
& \left.\delta \eta\right|_{\text {lat }} \stackrel{\frac{1}{a_{s}} \gg m, \gamma}{\approx} \frac{T}{\gamma} \int_{\mathbf{p}} \frac{\tilde{p}_{x}^{2} \tilde{p}_{y}^{2}}{\tilde{p}^{4}}=\frac{T \beta}{3 \gamma a_{s}^{3}}, \\
& \beta \equiv \int_{\mathbf{p}} \frac{a_{s}^{3}}{4 \sum_{i} \sin ^{2}\left(\frac{a_{s} p_{i}}{2}\right)}=\Gamma^{2}\left(\frac{1}{24}\right) \Gamma^{2}\left(\frac{11}{24}\right) \frac{\sqrt{3}-1}{192 \pi^{3}},
\end{aligned}
$$

where $\tilde{p}_{i} \equiv \frac{2}{a_{s}} \sin \left(\frac{a_{s} p_{i}}{2}\right)$ are lattice momenta and the integration is carried out over the first Brillouin zone.

The integral in Eq. (2.20) also has a finite part, which can be determined with dimensional regularization in $d$ spatial dimensions:

$\left.\delta \eta\right|_{\text {fin }}=\frac{T m^{d} \Gamma(2-d / 2)}{\gamma(4 \pi)^{d / 2} d(d-2)}\left(1-\frac{\gamma^{2} d}{4 m^{2}}\right)$.

Setting $d=3$ and $\gamma \sim m \sim \alpha^{2} T$, so that we are safely in the hydrodynamic regime, this is parametrically a very subleading contribution, $\left.\delta \eta\right|_{\text {fin }} \ll T^{3} / \alpha^{2}$. (We note that Eq. (2.24) 
becomes negative in the overdamped regime $\gamma \gtrsim m$, but this is of no concern, given that the full result from Eq. (2.20) remains positive.)

That Eqs. (2.20)-(2.24) diverge as $\gamma \ll m$, is familiar from other weakly coupled systems [15] and from the contribution of hydrodynamic fluctuations [16].

\section{Conclusions}

It seems conceptually attractive to incorporate scalar fluctuations into hydrodynamic simulations of cosmological phase transition dynamics. To begin with, this is theoretically necessary for respecting the fluctuation-dissipation theorem at a late time when the order parameter is approaching thermal equilibrium. In addition, thermal fluctuations would in principle lead to automatic bubble nucleations, even if in practice multicanonical simulations are needed for studying these rare events with their proper weights [17]. Fluctuations may also induce a first order phase transition [18], even if this would not happen with a scalar field alone. Finally, fluctuations would help in probing the instability of growing bubbles [19].

The practical inclusion of hydrodynamic fluctuations leads to powerlike ultraviolet divergences. In the regime of linear perturbations, the contribution from scalar fluctuations is cubically divergent in the formal continuum limit (cf. Eq. (2.21)), whereas that from normal hydrodynamic fluctuations is linearly divergent (cf. Eq. (A.18)). There is perturbative evidence that a cutoff-independent framework may be obtained by treating shear and bulk viscosities as "bare" parameters, and introducing counterterms for all possible thermodynamic functions, even if this leads to a rather complicated framework (cf. Ref. [20] for recent work and references). On a lattice, the loss of rotational symmetry may also become a concern $[11,12]$. However, turning the tables, a "bare simulation" would yield a well-predicted shear viscosity, cf. Eq. (2.22), and a corresponding contribution to the differential gravitational wave production rate at late times, cf. Eq. (2.15). The amplitude of this component can be tuned at will by changing the lattice spacing $a_{s}$ or the amplitude $\Omega$ of the noise auto-correlator. If the value of $\Omega$ deviates from that in Eq. (2.12), the resulting $\eta$ scales as $\Omega^{2} /(2 \gamma T)^{2}$ relative to Eq. (2.22). This behaviour of the overall magnitude, together with a corresponding spectral shape, are worth testing as a clean calibration of the measurement algorithm.

Acknowledgements This work was supported by the Swiss National Science Foundation (SNF) under Grant 200020-168988.

Open Access This article is distributed under the terms of the Creative Commons Attribution 4.0 International License (http://creativecomm ons.org/licenses/by/4.0/), which permits unrestricted use, distribution, and reproduction in any medium, provided you give appropriate credit to the original author(s) and the source, provide a link to the Creative Commons license, and indicate if changes were made.

Funded by SCOAP ${ }^{3}$.

\section{Appendix A: Contribution from normal hydrodynamic fluctuations}

For completeness and comparison with the scalar field case, we review here the contribution of normal hydrodynamic fluctuations to shear viscosity $[16,20]$.

In terms of the fundamental theory, we are considering a density matrix parametrized by a flow velocity $u^{\mu}$ and a temperature $T$ :

$\hat{\rho} \equiv \frac{1}{Z} \exp \left(-\frac{\hat{H} u^{0}-\hat{K}^{i} u^{i}}{T}\right)$,

where $\hat{H}$ is the Hamiltonian, $\hat{K}^{i}$ is the momentum operator, $u_{\mu} u^{\mu}=-1$, and $Z$ is chosen so that $\operatorname{Tr} \hat{\rho}=1$. Factoring out $u^{0} / T$, the thermodynamic pressure is defined as

$p\left(\frac{T}{u^{0}}, \frac{\mathbf{u}}{u^{0}}\right) \equiv \lim _{V \rightarrow \infty} \frac{T \ln Z}{u^{0} V}$,

where $V$ is the volume. Poincaré invariance implies that (cf. Ref. [21] and references therein)

$p\left(\frac{T}{u^{0}}, \frac{\mathbf{u}}{u^{0}}\right)=p(T, \mathbf{0}) \equiv p(T)$.

From this relation it can be shown that

$\lim _{V \rightarrow \infty} \frac{\langle\hat{H}\rangle}{V}=T \partial_{T} p-p \equiv e$,

$\lim _{V \rightarrow \infty} \frac{\left\langle\hat{K}^{i}\right\rangle}{V}=u^{0} u^{i} T \partial_{T} p \equiv u^{0} u^{i} w$,

where $e$ is the energy and $w$ the enthalpy density. These expectation values appear as parts of $T_{\text {ideal }}^{\mu \nu}=p g^{\mu \nu}+w u^{\mu} u^{\nu}$. The "susceptibility" related to $\hat{K}^{i}$ becomes

$\left.\lim _{V \rightarrow \infty} \frac{\left\langle\hat{K}^{i} \hat{K}^{j}\right\rangle}{V}\right|_{u^{i}=0}=T w \delta^{i j}$.

Let us now assume that $T$ and $u^{\mu}$ are not constant but vary slowly, and expand the expectation value of the energymomentum tensor to first order in gradients. Following Ref. [8], small variations cannot be distinguished from occasional long-wavelength thermal fluctuations $\left(S^{\mu \nu}\right)$, which must therefore be added as ingredients:

$$
\begin{aligned}
T^{\mu \nu}= & T_{\text {ideal }}^{\mu \nu}-\eta \Delta^{\mu \rho} \Delta^{\nu \sigma}\left(u_{\rho ; \sigma}+u_{\sigma ; \rho}-\frac{2 g_{\rho \sigma}}{d} u_{; \gamma}^{\gamma}\right) \\
& -\zeta \Delta^{\mu \nu} u_{; \gamma}^{\gamma}+S^{\mu \nu},
\end{aligned}
$$


where $\Delta^{\mu \nu} \equiv g^{\mu \nu}+u^{\mu} u^{\nu}$ is a projector onto directions orthogonal to $u^{\mu}$, and $\eta, \zeta$ are the shear and bulk viscosities. The noise correlator takes the form $[8,22]$

$$
\begin{aligned}
& \left\langle S^{\mu \nu}(\mathcal{X}) S^{\rho \sigma}(\mathcal{Y})\right\rangle=2 T\left[\eta\left(\Delta^{\mu \rho} \Delta^{\nu \sigma}+\Delta^{\mu \sigma} \Delta^{\nu \rho}\right)\right. \\
& \left.+\left(\zeta-\frac{2 \eta}{d}\right) \Delta^{\mu \nu} \Delta^{\rho \sigma}\right] \frac{\delta(\mathcal{X}-\mathcal{Y})}{\sqrt{-\operatorname{det} g_{\mu \nu}}} .
\end{aligned}
$$

We now restrict ourselves to flat spacetime and consider small perturbations of $T$ und $u^{i}$ around the equilibrium values $T_{0}$ and 0 , respectively. Small (non-relativistic) velocity fluctuations are denoted by $v^{i}$, and equilibrium values by $p_{0}, e_{0}, w_{0}, \eta_{0}, \zeta_{0}$, etc. It is helpful to go over into Fourier space, $f(\mathcal{X})=\int_{\mathcal{P}} e^{i \mathcal{P} \cdot \mathcal{X}} f(\mathcal{P})$, where $\mathcal{P} \equiv(\omega, \mathbf{p})$, and we also define $p \equiv|\mathbf{p}|$. Putting the terms originating from $S^{\mu v}$ on the right-hand side of the equation, defining

$\xi^{\mu}(\mathcal{P}) \equiv \frac{\mathcal{P}_{\nu} S^{\mu \nu}(\mathcal{P})}{w_{0}}$,

and denoting

$$
\begin{aligned}
\theta & \equiv \ln \left(\frac{T}{T_{0}}\right), \quad c_{s}^{2} \equiv \frac{\partial p_{0}}{\partial e_{0}}, \\
\bar{\eta}_{1} & \equiv \frac{\zeta_{0}+\frac{2(d-1)}{d} \eta_{0}}{w_{0}}, \quad \bar{\eta}_{2} \equiv \frac{\eta_{0}}{w_{0}},
\end{aligned}
$$

energy-momentum conservation $\partial_{\mu} T^{\mu \nu}=0$ implies that the temperature and velocity fluctuations are given by

$$
\begin{aligned}
\theta(\mathcal{P}) & =\frac{c_{s}^{2}\left[\left(\omega+i \bar{\eta}_{1} p^{2}\right) \xi^{0}(\mathcal{P})+p^{i} \xi^{i}(\mathcal{P})\right]}{\omega^{2}+i \bar{\eta}_{1} \omega p^{2}-c_{s}^{2} p^{2}}, \\
v^{i}(\mathcal{P}) & =\frac{p^{i}\left[c_{s}^{2} \xi^{0}(\mathcal{P})+\frac{\omega p^{j}}{p^{2}} \xi^{j}(\mathcal{P})\right]}{\omega^{2}+i \bar{\eta}_{1} \omega p^{2}-c_{s}^{2} p^{2}}+\frac{\left(\delta^{i j}-\frac{p^{i} p^{j}}{p^{2}}\right) \xi^{j}(\mathcal{P})}{\omega+i \bar{\eta}_{2} p^{2}} .
\end{aligned}
$$

These are the analogues of Eq. (2.8). Inserting Eq. (A.9), evaluating the thermal average according to Eq. (A.8), and noting that to leading order in $v^{i}$ only $S^{i j}$ contributes, the velocity correlator becomes (cf. e.g. Ref. [23])

$$
\begin{aligned}
\left\langle v^{i}(\mathcal{P}) v^{j}(\mathcal{Q})\right\rangle= & \delta(\mathcal{P}+\mathcal{Q}) G^{i j}(\mathcal{P}), \\
G^{i j}(\mathcal{P})= & \frac{2 T_{0}}{w_{0}}\left[\frac{\bar{\eta}_{1} \omega^{2} p^{i} p^{j}}{\left(\omega^{2}-c_{s}^{2} p^{2}\right)^{2}+\bar{\eta}_{1}^{2} \omega^{2} p^{4}}\right. \\
& \left.+\frac{\bar{\eta}_{2}\left(p^{2} \delta^{i j}-p^{i} p^{j}\right)}{\omega^{2}+\bar{\eta}_{2}^{2} p^{4}}\right] .
\end{aligned}
$$

Here $\delta(\mathcal{P}+\mathcal{Q}) \equiv(2 \pi)^{D} \delta^{(D)}(\mathcal{P}+\mathcal{Q})$ and $D \equiv d+1$. These are the analogues of Eq. (2.9).

It is useful to crosscheck that Eq. (A.15) reproduces the susceptibility from Eq. (A.6). To leading order in velocities, the components $S^{0 i}$ have a vanishing correlator. Therefore, from Eq. (A.7), $T^{0 i} \simeq w_{0} v^{i}$, and $\left\langle T^{0 i}(\mathcal{P}) T^{0 j}(\mathcal{Q})\right\rangle$ is directly proportional to Eq. (A.15). The equal-time correlator relevant for Eq. (A.6) can be obtained by integrating Eq. (A.15) over the frequency, $\int \frac{\mathrm{d} \omega}{2 \pi}$. Thereby we reproduce Eq. (A.6) in $d$ spatial dimensions.

We now move on to the correlator in Eq. (2.13). From Eq. (A.7),

$T^{x y} \approx w_{0} v^{x} v^{y}-\eta_{0}\left(\partial^{x} v^{y}+\partial^{y} v^{x}\right)+S^{x y}$.

According to Eq. (A.8), the noise part gives $\left\langle S^{x y}(\mathcal{K}) S^{x y}(\mathcal{Q})\right\rangle$ $=2 \eta_{0} T_{0} \delta(\mathcal{K}+\mathcal{Q})$, as expected from Eq. (2.14). In momentum space, the middle term vanishes for $\mathcal{K}=\left(k^{0}, k \mathbf{e}_{z}\right)$ as is relevant for Eq. (2.13). Following Ref. [16], we consider the 1-loop contribution from $w_{0} v^{x} v^{y}$ :

$$
\begin{aligned}
& 2 T_{0} \delta \eta_{0} \\
& \equiv \frac{w_{0}^{2}}{\delta(\mathcal{K}+\mathcal{Q})} \int_{\mathcal{P}, \mathcal{R}}\left\langle v^{x}(\mathcal{P}) v^{y}(\mathcal{K}-\mathcal{P}) v^{x}(\mathcal{R}) v^{y}(\mathcal{Q}-\mathcal{R})\right\rangle \\
& =w_{0}^{2} \int_{\mathcal{P}}\left[G^{x x}(\mathcal{P}) G^{y y}(\mathcal{K}-\mathcal{P})+G^{x y}(\mathcal{P}) G^{y x}(\mathcal{K}-\mathcal{P})\right]
\end{aligned}
$$

where we inserted Eq. (A.14). Substituting Eq. (A.15), integrating over $\omega$, and setting $\mathcal{K} \rightarrow 0$ as is sufficient according to Eq. (2.14), we obtain

$$
\begin{aligned}
& \lim _{\mathcal{K} \rightarrow 0} \delta \eta_{0}=2 T_{0} \int_{\mathbf{p}}\left\{\frac{p_{x}^{2} p_{y}^{2}}{2 \bar{\eta}_{1} p^{6}}+\frac{p^{4}-2 p^{2} p_{x}^{2}+2 p_{x}^{2} p_{y}^{2}}{4 \bar{\eta}_{2} p^{6}}\right. \\
&\left.+\frac{\bar{\eta}_{2}\left(p^{2} p_{x}^{2}-2 p_{x}^{2} p_{y}^{2}\right)}{\bar{\eta}_{2}\left(\bar{\eta}_{1}+\bar{\eta}_{2}\right) p^{6}+c_{s}^{2} p^{4}}\right\} \\
&=\frac{T_{0}}{d+2} \int_{\mathbf{p}}\left\{\frac{1}{\bar{\eta}_{1} p^{2} d}+\frac{d^{2}-2}{2 \bar{\eta}_{2} p^{2} d}\right. \\
&\left.+\frac{2 \bar{\eta}_{2}}{\bar{\eta}_{2}\left(\bar{\eta}_{1}+\bar{\eta}_{2}\right) p^{2}+c_{s}^{2}}\right\},
\end{aligned}
$$

where we made use of rotational symmetry to write $\int_{\mathbf{p}} p_{i} p_{j} p_{k} p_{l} \phi\left(p^{2}\right)=\frac{\delta_{i j} \delta_{k l}+\delta_{i k} \delta_{j l}+\delta_{i l} \delta_{j k}}{d(d+2)} \int_{\mathbf{p}} p^{4} \phi\left(p^{2}\right)$ and $\int_{\mathbf{p}} p_{i} p_{j} \phi\left(p^{2}\right)=\frac{\delta_{i j}}{d} \int_{\mathbf{p}} p^{2} \phi\left(p^{2}\right)$. Upon setting $d=3$, $\int_{\mathbf{p}} \frac{1}{p^{2}}=\frac{\Lambda}{2 \pi^{2}}$, and omitting the last term, this agrees with refs. $[16,20]$. The last term was omitted because at small $p$ it is suppressed by $\sim \bar{\eta}_{i}^{2} p^{2} / c_{s}^{2}$ compared with the other terms. In the continuum limit of a hydrodynamic simulation it should, however, be included as $2 /\left[\left(\bar{\eta}_{1}+\bar{\eta}_{2}\right) p^{2}\right]$.

\section{References}

1. C. Caprini et al., Science with the space-based interferometer eLISA. II: gravitational waves from cosmological phase transitions. JCAP 04, 001 (2016). arXiv:1512.06239

2. M. Hindmarsh, S.J. Huber, K. Rummukainen, D.J. Weir, Shape of the acoustic gravitational wave power spectrum from a first order phase transition. Phys. Rev. D 96, 103520 (2017). arXiv: 1704.05871 
3. J. Ghiglieri, M. Laine, Gravitational wave background from Standard Model physics: qualitative features. JCAP 07, 022 (2015). arXiv: 1504.02569

4. D. Bödeker, G.D. Moore, Electroweak bubble wall speed limit. JCAP 05, 025 (2017). arXiv:1703.08215

5. J. Ignatius, K. Kajantie, H. Kurki-Suonio, M. Laine, The growth of bubbles in cosmological phase transitions. Phys. Rev. D 49, 3854 (1994). arXiv:astro-ph/9309059

6. L.D. Landau, E.M. Lifshitz, Fluid Mechanics (ButterworthHeinemann, Oxford, 1987)

7. D. Bödeker, Moduli decay in the hot early Universe. JCAP 06, 027 (2006). arXiv:hep-ph/0605030

8. E.M. Lifshitz, L.P. Pitaevskii, Statistical Physics, Part 2, \$88-89 (Butterworth-Heinemann, Oxford, 1980)

9. S. Caron-Huot, Hard thermal loops in the real-time formalism. JHEP 04, 004 (2009). arXiv:0710.5726

10. G.D. Moore, K.A. Sohrabi, Kubo formulae for second-order hydrodynamic coefficients. Phys. Rev. Lett. 106, 122302 (2011). arXiv: 1007.5333

11. D. Bödeker, L.D. McLerran, A.V. Smilga, Really computing nonperturbative real time correlation functions. Phys. Rev. D 52, 4675 (1995). arXiv:hep-th/9504123

12. P.B. Arnold, Hot B violation, the lattice, and hard thermal loops. Phys. Rev. D 55, 7781 (1997). arXiv:hep-ph/9701393

13. G.N. Watson, Three triple integrals. Q. J. Math. 10, 266 (1939)

14. M.L. Glasser, J. Boersma, Exact values for the cubic lattice Green functions. J. Phys. A Math. Gen. 33, 5017 (2000)

15. S. Jeon, Hydrodynamic transport coefficients in relativistic scalar field theory. Phys. Rev. D 52, 3591 (1995). arXiv:hep-ph/9409250
16. P. Kovtun, G.D. Moore, P. Romatschke, The stickiness of sound: an absolute lower limit on viscosity and the breakdown of second order relativistic hydrodynamics. Phys. Rev. D 84, 025006 (2011). arXiv: 1104.1586

17. G.D. Moore, K. Rummukainen, Electroweak bubble nucleation, nonperturbatively. Phys. Rev. D 63, 045002 (2001). arXiv:hep-ph/0009132

18. B.I. Halperin, T.C. Lubensky, S.-K. Ma, First-Order Phase Transitions in Superconductors and Smectic- $A$ Liquid Crystals. Phys. Rev. Lett. 32, 292 (1974)

19. P.Y. Huet, K. Kajantie, R.G. Leigh, B.H. Liu, L.D. McLerran, Hydrodynamic stability analysis of burning bubbles in electroweak theory and in QCD. Phys. Rev. D 48, 2477 (1993). arXiv:hep-ph/9212224

20. Y. Akamatsu, A. Mazeliauskas, D. Teaney, Bulk viscosity from hydrodynamic fluctuations with relativistic hydro-kinetic theory. Phys. Rev. C 97, 024902 (2018). arXiv:1708.05657

21. L. Giusti, H.B. Meyer, Implications of Poincaré symmetry for thermal field theories in finite-volume. JHEP 01, 140 (2013). arXiv:1211.6669

22. J.I. Kapusta, B. Müller, M. Stephanov, Relativistic theory of hydrodynamic fluctuations with applications to heavy ion collisions. Phys. Rev. C 85, 054906 (2012). arXiv:1112.6405

23. P. Kovtun, L.G. Yaffe, Hydrodynamic fluctuations, long time tails, and supersymmetry. Phys. Rev. D 68, 025007 (2003). arXiv:hep-th/0303010 